\title{
Diseño de un seguro indexado para la
cobertura del riesgo de sequía hidrológica en la agricultura de regadío
}

\author{
Design of an index-based insurance for \\ hedging the risk of hydrological drought \\ in irrigated agriculture
}

José Antonio Gómez-Limón Universidad de Córdoba Córdoba, España jglimon@uco.es

María Dolores Guerrero-Baena Universidad de Córdoba Córdoba, España dolores.guerrero@uco.es

Resumen - En España, la agricultura de regadío es especialmente vulnerable al riesgo de sequía hidrológica, circunstancia que, en la práctica, conlleva que las dotaciones de agua que reciben los agricultores sean, en ocasiones, insuficientes para atender las necesidades de sus cultivos. Cuando esto ocurre, se produce el denominado fallo de garantía de suministro, que tiene importantes consecuencias negativas sobre la actividad agraria. Al objeto de minimizar los efectos negativos del 'fallo de garantía de suministro', en este trabajo se propone un seguro de sequía hidrológica para regadío indexado a la variable 'stock de agua disponible en los embalses de regulación', como nuevo instrumento de gestión de este riesgo. Se espera que esta propuesta, una vez transferida a la práctica, pueda contribuir a mejorar la gestión del riesgo de sequía en los sistemas agrarios de regadío, así como a garantizar la sostenibilidad y resiliencia de estos sistemas ante el progresivo impacto del cambio climático.

Abstract - In Spain, irrigated agriculture is especially vulnerable to the risk of hydrological drought. In practice, this circumstance implies that sometimes the water allotments received by farmers are insufficient to meet crops water needs, the so-called 'water supply gap', involving important negative consequences on farming activity. In order to minimize the negative effects of water supply gap, an indexbased drought insurance for irrigation is proposed, linked to the variable 'stock of water available in regulation reservoirs', as a new risk management instrument. It is expected that this proposal, once implemented into the real world, will be able to improve the management of drought risk in irrigated systems, as well as guarantying the sustainability and resilience of these systems under the increasing impact of climate change.

Palabras clave: Agua para riego, Cambio climático, Garantía de suministro, Seguro agrario, España

Keywords: Water for irrigation, Climate change, Water supply reliability, Agricultural insurance, Spain 


\section{INTRODUCCIÓN ${ }^{1}$}

La actividad agraria se caracteriza por su elevada exposición al riesgo ${ }^{2}$, esto es, a múltiples eventos adversos que pueden afectar negativamente a los ingresos y al bienestar del agricultor. De todos los tipos de riesgo a los que está sometido este sector, destacan los riesgos de producción derivados de factores climatológicos, tales como el granizo, las heladas o las sequías, que inciden sobre la cantidad y calidad de la producción agraria; y los riesgos de mercado, que tienen su origen en las fluctuaciones de los precios de los productos e insumos agrarios.

En España, los riesgos de producción derivados de factores climatológicos son más relevantes para los agricultores que los riesgos de mercado ${ }^{3}$. La razón estriba fundamentalmente en el clima mediterráneo de la mayoría de las regiones agrarias españolas, que se caracteriza por un patrón irregular de precipitaciones y por la elevada frecuencia de eventos climatológicos extremos. De entre todos estos eventos extremos, destaca la sequía como principal riesgo sistémico. La adecuada conceptualización de la sequía, no obstante, exige diferenciar varios tipos: por un lado, se presenta una situación de sequía meteorológica cuando el nivel de lluvias es menor al promedio, pero tal situación no provoca impactos significativos; por otro lado, se produce la sequía agrícola cuando la falta de precipitaciones genera un déficit de humedad en la zona radicular del suelo que impacta negativamente sobre el crecimiento de la vegetación, afectando a los cultivos y pastos de secano; además, el agravamiento de la falta de lluvias puede derivar en sequía hidrológica, que ocurre cuando los caudales circulantes por los cursos de agua y los volúmenes embalsados se sitúan por debajo de lo normal.

En el caso de la agricultura de regadío, el riesgo de sequía al que se enfrenta es el de la sequía hidrológica, puesto que su suministro de agua depende directamente del agua disponible en los embalses de regulación. Efectivamente, los regantes en España cuentan con concesiones administrativas o derechos de agua normalmente equivalentes a la cantidad de agua necesaria para cubrir por completo las necesidades hídricas de sus cultivos. Sin embargo, en los años en los que el agua almacenada en los embalses es inferior al total de los derechos otorgados (situación de sequía hidrológica), se hace necesario imponer restricciones o limitaciones a las dotaciones anuales (cantidades de agua anuales para riego a disposición del agricultor) siguiendo un criterio proporcional. Así, los regantes españoles se enfrentan a una elevada variabilidad interanual en sus dotaciones de agua para riego que, en ocasiones, pueden situarse muy por debajo de lo establecido en sus concesiones, e incluso llegar a ser nulas. Cuando esto ocurre (dotación menor a la concesión) se produce el denominado "fallo en la garantía

\footnotetext{
1 Este trabajo ha sido financiado por el Ministerio de Economía y Competitividad (MINECO) y el Fondo Europeo de Desarrollo Regional (FEDER) a través del proyecto de investigación MERCAGUA (AGL2013-48080-C2-1-R). Los autores agradecen las sugerencias e indicaciones realizadas por los evaluadores anónimos, que han permitido la mejora del documento durante el proceso de revisión científica del mismo.

2 Moschini y Hennessy, 2001. OECD, 2011.

${ }^{3}$ Antón y Kimura, 2011. OECD, 2011.
}

de suministro", situación que genera una pérdida de producción y de renta del agricultor.

La preocupación de los regantes españoles (principalmente, del sur y este peninsular) por las consecuencias sobre su bienestar de un posible fallo en la garantía de suministro se ha acrecentado exponencialmente en los últimos años debido al actual escenario de cambio climático. Las negativas predicciones sobre las precipitaciones (menores disponibilidades de agua) y la previsible subida de las temperaturas (mayores necesidades hídricas de los cultivos) podrían llegar incluso a poner en peligro la viabilidad de la agricultura de regadío en muchas de estas zonas. Por ello, en los últimos años se ha planteado la necesidad de diseñar nuevos instrumentos de gestión del riesgo que puedan ser adoptados por los regantes para minimizar los impactos negativos de las contingencias hidrológicas ${ }^{4}$. Entre los potenciales instrumentos sugeridos para ello destaca el seguro, que se ha señalado como una herramienta eficiente para amortiguar los efectos económicos ocasionados por la sequía hidrológica en el regadío ${ }^{5}$.

A pesar de que el seguro agrario se encuentra muy desarrollado en España, no existe actualmente cobertura para el fallo en la garantía de suministro de agua en la agricultura de regadío. En este contexto, el objetivo principal de este trabajo es contribuir al incipiente debate académico sobre la forma de superar esta carencia, proponiendo un diseño de seguro de sequía hidrológica que resulte técnicamente factible (supere los problemas del diseño tradicional del seguro agrario que impide actualmente que se cubra esta contingencia) y comercialmente viable (coste actuarial del mismo inferior a la disposición a pagar de los asegurados). Para ello, se propone como primer objetivo específico realizar una exhaustiva revisión de todos los factores que limitan actualmente el desarrollo de este tipo de seguro, así como de las fórmulas disponibles para disminuir el alcance de tales limitaciones. Asimismo, como segundo objetivo específico se propone hacer una revisión crítica de los trabajos previos que han planteado contratos de seguro para cubrir el riesgo de sequía hidrológica, la cual permitirá fundamentar la discusión acerca de la concreción de los elementos que deben caracterizar este instrumento de gestión del riesgo para que pueda ser técnica y comercialmente viable. Finalmente, el tercer objetivo específico de este trabajo es proponer un nuevo seguro de sequía hidrológica adaptado a las condiciones del regadío en España, diseñado como un seguro indexado basado en la variable "stock de agua disponible en los embalses de regulación”.

\section{EL RIESGO DE FALLO DE SUMINISTRO DE AGUA DE RIEGO}

Como se ha comentado, en España el sector agrario se encuentra especialmente expuesto a riesgos de producción derivados de fenómenos climatológicos. Esta exposición al riesgo es especialmente importante en las regiones del sur y este peninsular, dadas las fuertes oscilaciones meteorológicas propias del clima mediterráneo y, en especial, debido a la gran variabilidad intra- e inter-anual

\footnotetext{
${ }^{4}$ Antón, 2009. Garrido y Gómez-Ramos, 2009. OECD, 2016. Riera, 2018.

${ }^{5}$ Rey et al., 2019.
} 
de las precipitaciones, lo que convierte a la agricultura de estas regiones en una actividad marcada por el riesgo de sequía. Además, este riesgo está aumentando en estas regiones debido al impacto del cambio climático ${ }^{6}$. En este sentido, y de acuerdo con el IPCC (2014), las predicciones para los próximos años en el caso de las regiones de clima mediterráneo y semiárido advierten que se producirá un decremento de las precipitaciones (sequía agrícola) y de la disponibilidad de agua (sequía hidrológica), así como un aumento progresivo de las temperaturas, circunstancias que incrementarán las necesidades de agua del regadío. Además, las predicciones para estas regiones señalan que los periodos de sequía serán cada vez más frecuentes e intensos.

La variabilidad de las precipitaciones se traduce en una fuerte variabilidad de los rendimientos de los sistemas agrarios de secano, donde la lluvia es la única fuente de agua. Los episodios de sequía afectan de manera igualmente negativa a los sistemas agrarios de regadío, si bien la intensidad de sus efectos está supeditada a la inercia de los sistemas hidráulicos de los que depende, según tengan una mayor o menor capacidad de almacenamiento de agua. En estos sistemas, las dotaciones de agua para el riego que reciben cada año los agricultores son aprobadas por el organismo de cuenca correspondiente, que decide el volumen de agua a percibir por cada zona regable en función de las disponibilidades existentes en los embalses. Esta dotación anual es posteriormente repartida entre los regantes de cada zona regable por la comunidad de regantes correspondiente, siguiendo un criterio igualmente proporcional. Así, tras un periodo de sequía meteorológica prolongado (por ejemplo, tras un otoño-invierno seco en el caso de un sistema de escasa capacidad de regulación hídrica, o tras varios años secos en sistemas más regulados), la sequía se convierte en hidrológica (déficit de recursos embalsados) y las dotaciones de riego aprobadas tienen que fijarse por debajo de la concesión administrativa, produciéndose así un "fallo de garantía de suministro".

Las consecuencias concretas del fallo de suministro en la agricultura de regadío se pueden clasificar según sean: económicas, sociales o ambientales. Por un lado, desde el punto de vista microeconómico, el fallo de suministro disminuye la renta del agricultor, pues este tendrá que ajustar la superficie regada y su plan de cultivos en función de la disponibilidad de agua. A nivel macroeconómico, esta situación produce una disminución de la producción y del valor añadido del sector agrario, así como de otros sectores productivos relacionados, como el sector de insumos agrícolas. Además, debido a la aversión al riesgo que caracteriza a la mayoría de los agricultores ${ }^{7}$, en situaciones de elevada incertidumbre acerca de la disponibilidad de agua para riego, la toma de decisiones productivas de los agricultores no resulta eficiente desde una perspectiva pública, en la medida que estos tienden a minorar el uso de insumos productivos ${ }^{8}$ y la realización de inversiones ${ }^{9}$, con la consiguiente merma en la producción agraria.

\footnotetext{
${ }^{6}$ OECD, 2011.

${ }^{7}$ Menapace et al., 2013.

${ }^{8}$ Beare et al., 1998.

${ }^{9}$ Marques et al., 2005.
}

Por otro lado, desde una perspectiva social, el fallo de suministro en el regadío provoca una disminución del empleo agrario, en la medida que una hectárea de regadío genera de media 3,8 veces más empleo que una de secano $^{10}$. Además, en los casos en los que este riesgo sea significativo, la incertidumbre hidrológica puede conllevar el abandono (o no implantación) de determinados cultivos especialmente sensibles a cortes en el suministro de riego, como son los cultivos frutales, normalmente muy intensivos en el uso de mano de obra.

Finalmente, caben señalar las consecuencias ambientales de los fallos de suministro, entre las que destaca la extracción ilegal de recursos hídricos (p. ej., extracción de aguas subterráneas) al objeto de sustituir parcialmente los recortes en las dotaciones, circunstancia que puede agravar los problemas de sobrexplotación de las masas de agua afectadas.

De todo lo comentado resulta obvio que, del conjunto de riesgos climatológicos, la incertidumbre acerca de la disponibilidad de agua para el riego constituye uno de los principales riesgos de producción a los que se enfrentan los agricultores de regadío españoles ${ }^{11}$. La estrategia tradicionalmente implementada para minimizar este riesgo de fallo en la garantía de suministro se ha basado, principalmente, en el desarrollo de políticas públicas de agua desde la perspectiva de la oferta, enfocadas a la construcción de infraestructuras de regulación tales como embalses, al objeto de captar y almacenar una mayor cantidad de agua. Sin embargo, estas políticas de oferta han sido superadas por la creciente demanda de agua, que a la postre han provocado una reducción en la garantía de suministro. A esta circunstancia hay que sumar las crecientes dificultades para incrementar la regulación hidráulica, dadas las limitaciones ambientales, cada vez más exigentes, impuestas a estas inversiones. Esta situación es especialmente evidente en las cuencas con mayor escasez de recursos (p. ej., zonas del sur y este de España), que se consideran hidrológicamente "cerradas" 12 , en la medida que cualquier nueva demanda de agua solo puede satisfacerse manteniendo la misma garantía si se reducen los derechos concesionales de otros usuarios.

El cierre de cuencas está provocando que tanto la comunidad científica como los decisores políticos exploren nuevos mecanismos o instrumentos de gestión hídrica para gestionar el riesgo de fallo en la garantía de suministro desde el punto de vista de la demanda ${ }^{13}$. Este tipo de instrumentos políticos tienen por objeto gestionar los actuales recursos hídricos disponibles para optimizar la eficiencia en el uso del agua y reducir la exposición al riesgo de fallo de suministro de los usuarios. Entre tales instrumentos, destacan la modernización del regadío ${ }^{14}$, la tarificación ${ }^{15}$, los mercados de agua ${ }^{16}$, los bancos de agua $^{17}$, los contratos de opción ${ }^{18}$ y los seguros de sequía

\footnotetext{
10 Gómez-Limón y Picazo-Tadeo, 2012.

11 Rey et al., 2016b.

12 Expósito y Berbel, 2017.

13 Lago et al., 2015.

14 Berbel y Gutiérrez-Martín, 2017.

15 Gallego-Ayala et al., 2011.

${ }^{16}$ Palomo-Hierro et al., 2015.

17 Montilla-López et al., 2016.

18 Rey et al., 2016a.
} 
hidrológica ${ }^{19}$. Sin embargo, estos instrumentos apenas se han implementado en la práctica en España, a excepción de la modernización de regadíos.

Para el conjunto de la agricultura, durante la última década se ha promovido la utilización del seguro agrario como instrumento para la gestión del riesgo al que se enfrenta el agricultor ${ }^{20}$. Con este seguro, los agricultores consiguen minimizar la incertidumbre acerca de los distintos sucesos negativos (de producción o de mercado) a los que se exponen sus explotaciones mediante la transferencia del riesgo a una compañía aseguradora ${ }^{21}$. En este sentido, la Organización para la Cooperación y el Desarrollo Económicos ${ }^{22}$ y la Comisión Europea ${ }^{23}$ sugieren, además, que es necesario ampliar la cobertura de riesgos hacia aquellos que en la actualidad no se contemplan en los sistemas aseguradores, como es el caso del fallo de la garantía de suministro de agua en regadío. Estas instituciones consideran, además, necesario el establecimiento de incentivos públicos que permitan la generalización del seguro agrario (incluyendo el de sequía hidrológica) como herramienta útil de gestión del riesgo, posibilitando que su oferta sea viable en términos financieros para las empresas aseguradoras, y el precio de las pólizas sea aceptable para los agricultores. Este apoyo público queda justificado, como se ha comentado previamente, por la contribución del seguro agrario a la mejora del bienestar social (mejora del nivel de producción agraria y minoración de impactos sociales y ambientales negativos asociados a los siniestros agrarios).

En estos términos, la finalidad de un seguro de sequía hidrológica para regadío sería conseguir garantizar al agricultor un nivel estable de renta en aquellos años en que su dotación de agua para riego se viera limitada como consecuencia de un periodo de sequía. De esta manera, este seguro amortiguaría los efectos económicos del fallo en la garantía de suministro, disminuyendo la incertidumbre del agricultor y permitiéndole la adopción de decisiones económicas más eficientes.

FACTORES QUE DIFICULTAN LA IMPLEMENTACIÓN DEL SEGURO DE SEQUÍA HIDROLÓGICA PARA EL REGADÍO

El Sistema Español de Seguros Agrarios Combinados (en adelante, SESAC), regulado mediante la Ley 87/1978, de 28 de diciembre, de Seguros Agrarios Combinados, se configura mediante un sistema mixto público-privado, siendo en la actualidad uno de los más avanzados y completos, no sólo de la Unión Europea, sino también de todo el mundo ${ }^{24}$. El SESAC contempla prácticamente todas las producciones agrícolas, siendo la cobertura del riesgo de producción derivado de cuestiones climatológicas muy elevada, dado que cubre tanto los riesgos puntuales (p. ej., el pedrisco o la helada), como los riesgos sistémicos (aquellos cuyos siniestros afectan simultáneamente a un gran número de asegurados, como pueden ser la sequía agrícola, aunque solo para cultivos de

\footnotetext{
19 Pérez-Blanco y Gómez, 2014.

${ }^{20}$ World Bank, 2005. OECD, 2016. EC, 2017b.

${ }^{21}$ Borch, 1990. Bardají et al., 2016.

22 OECD, 2016.

23 EC, 2017a.

24 Burgaz, 1995. Burgaz y Pérez-Morales, 1996. Antón y Kimura, 2011. Meuwissen et al., 2018.
}

secano $)^{25}$. La madurez del SESAC se evidencia en los datos de su implantación; en el año 2017 se contrataron más de 234 mil pólizas, que cubrían 13,8 millones de hectáreas (36,9\% de la superficie de cultivos), asegurando un capital de $9.928 \mathrm{M} €$ (34,6\% del valor de la producción agrícola $)^{26}$. Buena parte de este éxito se debe al abaratamiento del seguro por las subvenciones concedidas por el Estado y las Comunidades Autónomas, que promedian el $40 \%$ del coste de las pólizas ${ }^{27}$.

A pesar de su desarrollo y amplia cobertura, el SESAC no contempla como riesgo asegurable el fallo en la garantía de suministro de agua en la agricultura de regadío. En este sentido, varios son los factores que dificultan su implementación y justifican que este tipo de seguro no se haya desarrollado todavía, ni en España, ni en la mayoría de los países con sistemas aseguradores avanzados $^{28}$. Entre estos factores destacan, en primer lugar, aquellos comunes al resto de líneas del seguro agrario:

a) El seguro de cobertura de la sequía hidrológica para regadío, al igual que el conjunto del seguro agrario, adolece del denominado problema del riesgo moral, relacionado con la asimetría en la información. Este problema se presenta cuando el asegurado, una vez contratado el seguro, influye intencionadamente mediante su comportamiento en el resultado final del mismo, sin que el asegurador tenga conocimiento cierto de este comportamiento $^{29}$. Este sería el caso, por ejemplo, de un agricultor de regadío que tuviera contratado un seguro de sequía hidrológica y que, en situaciones de restricciones parciales en el uso del agua (fallo parcial en la garantía de suministro), no empleara la dotación concedida para regar, al objeto de ahorrar costes y, a la vez, poder cobrar una mayor indemnización por siniestro.

b) Otro problema relacionado con la asimetría en la información es el problema de la selección adversa, que consiste en que aquellos agricultores con más probabilidades de sufrir siniestros contratan en mayor medida pólizas de seguro que aquellos que tienen menos probabilidades de sufrir estas contingencias. Así, como la fijación de las primas se realiza para cada línea de seguro considerando el conjunto de la siniestralidad de todos los agricultores asegurados, aquellos productores que tienen menos probabilidades de sufrir siniestros (esperanza de la indemnización inferior al coste de la prima) no estarán tan motivados a contratar el seguro como aquellos con mayores probabilidades (esperanza de la indemnización mayor al coste de la prima), que lo percibirán como un instrumento muy atractivo. De esta manera, se produce un proceso de “autoselección” donde los suscriptores del seguro

\footnotetext{
25 Machetti, 2015.

26 Agroseguro, 2018.

27 Ídem.

${ }^{28}$ Pérez-Blanco y Gómez, 2013, 2014.

${ }^{29}$ Mishra et al., 2005.
} 
serían cada vez los más propensos a sufrir siniestros $^{30}$

Ambos problemas generan una situación de desequilibrio actuarial para el asegurador (pagos por siniestros mayores a los cobros por las primas), que conduce inevitablemente a un encarecimiento progresivo del seguro y a la ineficiencia de este instrumento ${ }^{31}$. Sin embargo, como se evidencia en las demás líneas de seguros agrarios, ambos problemas pueden limitarse aplicando las medidas oportunas. Para minimizar los problemas de asimetría de información habría que:

a) Segmentar a los agricultores en función del nivel de riesgo de fallo de suministro (p. ej., a nivel de subcuenca hidrográfica o sistema de explotación hidráulico) para aplicarles primas adaptadas al riesgo real de cada uno de ellos.

b) Implantar sistemas "bonus-malus" que corrijan individualmente la prima en función del comportamiento del agricultor.

c) Establecer franquicias deducibles.

d) Aplicar reglas de peritación que auditen el comportamiento de los agricultores en caso de siniestro.

Lógicamente, todas estas medidas encarecen el diseño y la administración del seguro, generando unos costes de transacción que se repercuten encareciendo la prima del seguro.

Además de los problemas anteriores, el diseño e implementación de un seguro de sequía hidrológica para el regadío presenta problemas específicos que requieren igualmente ser solventados para viabilizar este tipo de seguro:

a) El carácter sistémico de la sequía hidrológica ${ }^{32}$, que implica que el riesgo de fallo de garantía de suministro afecta a un gran número de agricultores de una misma cuenca, comprometiendo la capacidad de pagar el conjunto de indemnizaciones por parte de las compañías de seguros, lo que puede condicionar la viabilidad de las empresas aseguradoras. Este problema se podría solucionar, por un lado, obligando a las empresas aseguradoras a dotar una gran cantidad de reservas y, por otro lado, mediante la utilización del reaseguro, medidas ambas que encarecerían igualmente el coste de las primas. En cualquier caso, el SESAC ha solventado convenientemente este problema, dado que incluye en su estructura un cuadro de coaseguro, según el cual varias compañías aseguradoras cubren los mismos riesgos al objeto de compartirlos y disminuir su exposición a los mismos, así como un programa de reaseguro público. Así, se garantiza la viabilidad de todo el sistema en caso de siniestros extremos ${ }^{33}$. Estas características del SESAC le permiten asegurar actualmente riesgos sistémicos, tales como la sequía en cultivos de secano, por lo que también cabría asegurar el riesgo de fallo de suministro en la agricultura de regadío.

b) En España, los regantes (potenciales asegurados) están representados en las comisiones de desembalse de los organismos de cuenca, órgano donde se proponen las dotaciones anuales de agua para riego. La posibilidad de que los propios asegurados puedan incidir en la probabilidad de ocurrencia de siniestros (dotaciones inferiores a los derechos concesionales), convierte el riesgo de fallo de suministro en no asegurable, dado que no puede garantizarse que su ocurrencia sea verdaderamente accidental. Este inconveniente, sin embargo, se ha minorado de manera importante desde la aprobación de los Planes Especiales de Sequía (PES). Tradicionalmente, los organismos de cuenca, a propuesta de las comisiones de desembalse, han contado con una discrecionalidad absoluta para tomar decisiones respecto de las dotaciones que se concedían a los regantes cada campaña de riego. Sin embargo, desde la aprobación de los PES en 2007, estos organismos están obligados a tomar tales decisiones siguiendo los protocolos de actuación establecidos al efecto, al menos teóricamente, puesto que en la práctica no siempre ha sido así. Efectivamente, estos planes, redactados conforme a las exigencias de la Directiva Marco de Agua, establecen un procedimiento técnico conocido de antemano para el reparto de los recursos disponibles basado en un sistema de escenarios posibles (normalidad, prealerta o escasez del recurso moderada, alerta o escasez severa, y emergencia o escasez grave). Estos planes establecen procedimientos formalizados de obligado cumplimiento para la fijación de las dotaciones anuales de agua, minimizando la arbitrariedad existente hasta ahora. En todo caso, cualquier propuesta que se haga para un seguro de sequía hidrológica deberá basarse en un procedimiento objetivo y no manipulable para la determinación de la ocurrencia de siniestros y de su intensidad, ambos factores clave en el cálculo de las indemnizaciones correspondientes. Como luego se comenta, los seguros indexados son una buena opción en este sentido.

c) La incertidumbre en relación con las posibles modificaciones, como consecuencia del cambio climático, de la función de distribución de probabilidad que caracteriza la variable dotación anual de agua de riego, necesaria en el cálculo actuarial de la prima del seguro. Estos desplazamientos de la función de distribución de probabilidad de la disponibilidad de agua pueden afectar a los estadísticos media y varianza. Como ya se ha comentado, los modelos climáticos prevén, aunque no de manera precisa, una diminución de la media de esta variable y un aumento de su varianza, cambios que incrementarían la prima del seguro de sequía hidrológica. Especialmente relevantes son los cambios en la media de la disponibilidad de agua,

\footnotetext{
30 Just et al., 1999.

31 Coble et al., 1997.

32 Skees et al., 2008.

${ }^{33}$ Bardají et al., 2016.
} 
pues este estadístico es el que determina en mayor medida la prima del seguro. Así, si la media de esta variable disminuye considerablemente, las primas que sería necesario aplicar serían muy elevadas, llegando incluso a imposibilitar la viabilidad económica del seguro. La incertidumbre de los aseguradores acerca de las posibles modificaciones en la función de distribución de la disponibilidad de agua puede solventarse añadiendo a la prima del seguro una carga adicional por ambigüedad, como única vía para posibilitar que las aseguradoras acepten asumir este riesgo ${ }^{34}$.

d) La existencia de diferentes fuentes de suministro de agua de riego genera igualmente un problema, dado que los fenómenos de sequía hidrológica no afectan por igual a todas ellas. Piénsese, por ejemplo, en un regante que cuenta con una concesión de agua superficial, pero que también puede acceder a fuentes de agua subterránea, agua regenerada o agua desalada. En este caso, la sequía hidrológica se manifestaría disminuyendo la dotación de agua superficial, pero el regante podría compensar este fallo de suministro empleando otras fuentes, como por ejemplo el agua desalada, totalmente segura. En estas circunstancias, el fallo de suministro del agua superficial no sería asegurable, dada la dificultad de cumplir con el principio indemnizatorio, por el que el seguro no debe suponer una fuente de enriquecimiento para el asegurado. Es probable que la ocurrencia de un fallo de suministro colocase en mejor situación al asegurado que si el siniestro no hubiera ocurrido, ya que la indemnización podría ser superior al sobrecoste que supone acudir a otras fuentes de suministro de agua. Este problema hace que sólo sea susceptible el aseguramiento de aquellos regantes que cuenten con una única fuente de suministro de agua y cuya disponibilidad de agua para riego sea fácil de verificar y controlar. Así, en la práctica, el seguro de sequía hidrológica resulta factible sólo para los regantes que cuenten con una concesión de agua superficial dotada anualmente por el organismo de cuenca como única fuente de suministro.

e) La legislación española permite la cesión temporal de derechos de agua, tanto a través de los contratos de cesión (mercados de agua) como mediante los centros de intercambio de derechos (bancos de agua) que pueden constituirse durante períodos de sequía $^{35}$. Esta circunstancia hace que el uso de agua por parte del regante en su propia explotación dependa de sus propias decisiones (si cede o no temporalmente sus derechos), y no de un fenómeno estocástico asegurable. Por este motivo, el seguro de sequía hidrológica no puede asegurar los daños sobre los cultivos por la falta de agua, ni la pérdida de renta por el cambio en el plan de cultivos consecuencia de la escasez,

\footnotetext{
34 Skees et al., 2008.

35 Palomo-Hierro y Gómez-Limón, 2013.
}

dado que ambas pérdidas pueden ser agravadas intencionadamente en caso de siniestro (fallo en la garantía de suministro) como consecuencia de la cesión voluntaria de derechos por parte del regante. Esta circunstancia obliga a que el seguro de sequía considere como capital asegurable el valor de la dotación de agua, y no el correspondiente a los rendimientos de los cultivos, como ocurre en la mayoría de las líneas del seguro agrario. Así, el riesgo que debe gestionar el seguro de sequía hidrológica debe ser el lucro cesante derivado del fallo en el suministro de agua de riego por la reducción de la dotación de agua concedida por el organismo de cuenca, con independencia del uso efectivo del agua (planes de cultivos y dosis de riego por cultivos).

f) La gestión plurianual de las reservas de agua de los sistemas de explotación hidráulicos con mayor regulación hidráulica dificulta correlacionar temporalmente la ocurrencia de los fenómenos que causan la sequía (disminución de la pluviometría y las escorrentías que alimentan los embalses) y sus efectos (recortes en las dotaciones de riego). Por ejemplo, existen numerosos sistemas de explotación hidráulicos en los cuales si a comienzos del año hidrológico (octubre) los embalses están a nivel "normal”, durante ese año, aunque haya sequía meteorológica e incluso agrícola, lo normal es que se puedan satisfacer todas las demandas de riego (nula probabilidad de fallo en la garantía de suministro). La sequía hidrológica en estos sistemas altamente regulados solo ocurriría si se sucedieran varios años de lluvias significativamente por debajo de la media. Esta circunstancia pone en duda la conveniencia de pólizas de seguro de sequía hidrológica anuales, como en el resto de las líneas del seguro agrario, en la medida que la probabilidad de siniestro en un año dado no es un fenómeno estadísticamente independiente, sino que está condicionada por la ocurrencia o no de siniestro los años anteriores (la probabilidad de que haya fallo de suministro no es la misma si el año comienza con un nivel de agua embalsada por encima o por debajo de la media histórica). Este hecho apunta a la necesidad de establecer condiciones en la contratación que fomenten la renovación de las pólizas año tras año, o bien, diseñar pólizas plurianuales.

Los problemas anteriores no son exclusivos del caso español, sino que son compartidos igualmente por muchos otros países, lo que explica que este tipo de seguro raramente se haya implementado. De hecho, tan sólo en EE.UU. existe este tipo de cobertura en la agricultura de regadío, dentro del denominado "seguro multi-riesgo contra pérdidas en cosechas” (MPCI, por sus siglas en inglés; Multiple-Peril Crop Insurance).

A pesar de todas estas dificultades, diversos trabajos académicos han tratado de buscar soluciones que posibiliten plantear un seguro de sequía hidrológica para regadío. Todas estas aportaciones permiten concretar una 
nueva propuesta de un diseño viable para este tipo de seguro para el caso del regadío en España.

TIPOLOGía DE LOS SEGUROS AGRARIOS: OPCIONES PARA EL SEGURO DE SEQUÍA HIDROLÓGICA PARA REGADÍO

Los sistemas de seguros agrarios pueden incorporar varios tipos de seguros. Una forma habitual de clasificarlos es atendiendo al carácter único o múltiple de los riesgos asegurados. Así, se puede diferenciar entre:

a) Seguro de riesgo único, por ejemplo, para cubrir el riesgo de granizo.

b) Seguro combinado, que contempla la cobertura de varios riesgos, como pueden ser el granizo y la helada.

c) Seguro de rendimientos, que cubre todos los riesgos climáticos que puedan incidir en la producción agraria, incluidos los riesgos sistémicos, como la sequía agrícola en secano.

Otra posible clasificación de los seguros atiende al modo de evaluar la pérdida producida en la explotación agraria como consecuencia de la contingencia asegurada. Así, se podría distinguir entre:

a) Seguros de valoración de daños mediante peritación en la propia explotación, denominados comúnmente "seguros tradicionales".

b) Seguros indexados, en los que la valoración del daño se realiza de manera indirecta a través de una variable o índice correlacionado con la contingencia cubierta. Entre los seguros indexados, se distinguen a su vez varios tipos ${ }^{36}$ :

- Seguros indexados de rendimientos o de ingresos, donde el índice se calcula como la media de los rendimientos o ingresos de una región, y las pérdidas por siniestro se fijan en función de ese índice.

- Seguros indexados indirectos, en los que el índice se elabora a través de una o varias variables exógenas a la explotación. Así, podría diferenciarse entre:

* Seguros indexados climáticos, que consideran variables tales como las precipitaciones o la temperatura.

* Seguros indexados agro-climáticos, que tienen en cuenta indicadores tales como la humedad de la tierra.

* Seguros indexados basados en imágenes de satélite, por ejemplo, para obtener índices de vegetación.

* Seguros indexados basados en otras variables, como por ejemplo, en la cantidad de agua almacenada en los embalses o los flujos entrantes en los mismos para un seguro de sequía hidrológica.

Aunque los seguros tradicionales de valoración de daños son los más extendidos, tanto a nivel español como internacional, los seguros indexados ofrecen una serie de ventajas que disminuyen considerablemente el coste de la prima comercial del seguro ${ }^{37}$ :

\footnotetext{
${ }^{36}$ Bielza et al., 2008.

${ }^{37}$ World Bank, 2005. Skees et al., 2008. Jensen y Barrett, 2017.
}

a) Al calcularse las indemnizaciones en función del valor de un índice objetivo y no manipulable, no es necesaria la peritación del daño en la propia explotación.

b) Existe una mayor transparencia en el cálculo de las indemnizaciones frente al seguro tradicional, sin que haya cabida a la arbitrariedad, por lo que se minimizan los costes relacionados con la resolución de conflictos.

c) Debido a que la información relativa al índice (por ejemplo, información sobre la variable precipitaciones) es la misma tanto para el asegurado como para el asegurador, el problema de la selección adversa disminuye.

d) Como el agricultor no tiene capacidad para influir mediante su comportamiento en el resultado del valor del índice, se elimina el problema de riesgo moral.

Sin embargo, como mayor inconveniente de los seguros indexados destaca la existencia del denominado "riesgo base"; esto es, el riesgo relacionado con la posible diferencia entre el valor de la indemnización basada en el índice y la pérdida real producida en la explotación. Podría suceder que un agricultor sufriera una determinada pérdida en su explotación pero que, en base al valor del índice, no le correspondiera recibir indemnización alguna, o que esta fuera inferior a la pérdida. Igualmente podría existir el caso contrario, por el cual un agricultor que no sufriera realmente daño fuese indemnizado por el seguro en base a dicho índice. Estas situaciones se producen si no hay una elevada correlación entre el índice y la pérdida real experimentada en la explotación. Teniendo en cuenta esta circunstancia, es evidente que la primera condición que debe cumplir una variable para ser considerada un índice en un seguro indexado es que exista una alta correlación de la variable con la pérdida sufrida en las explotaciones agrarias, al objeto de disminuir el riesgo base. Otras condiciones relevantes que debe reunir todo índice para ser la base de un seguro indexado son las siguientes $^{38}$ :

a) La metodología de cálculo del índice debe estar disponible a todos los potenciales asegurados.

b) Los valores que adopte el índice deben ser totalmente objetivos y no manipulables, debiendo hacerse públicos regularmente mediante canales de difusión adecuados.

c) Deben existir registros históricos de las variables necesarios para calcular el índice, al objeto de que las compañías aseguradoras puedan realizar los pertinentes estudios actuariales en base al índice propuesto.

Finalmente, cabe señalar que los seguros agrarios también pueden clasificarse, en función del papel de los poderes públicos, entre privados y públicos ${ }^{39}$. Cuando el rol de las administraciones es puramente regulatorio y de defensa de la competencia, estamos ante un seguro agrario privado. Cuando el seguro agrario forma parte de la política agraria, el Estado crea un marco de regulación del sector diferente, que trata de promocionar este instrumento de gestión del riesgo mediante subvenciones

\footnotetext{
38 World Bank, 2011.

39 Bardají et al., 2016.
} 
y reaseguro público de los riesgos cubiertos, dados los beneficios sociales derivados de su implementación ${ }^{40}$. En este contexto es donde se ha desarrollado el SESAC como sistema de seguro mixto público-privado ${ }^{41}$.

\section{PROPUESTAS DE UN SEGURO DE SEQUíA HIDROLÓGICA PARA REGADÍO} DESDE EL ÁMBITO ACADÉMICO

Diferentes estudios han detectado el elevado interés de los regantes españoles en disminuir la incertidumbre asociada a la elevada variabilidad de sus dotaciones de agua para riego ${ }^{42}$. Este interés se materializa, en la práctica, en la disposición a pagar (DAP) por conseguir disminuir esta incertidumbre. Estos estudios evidencian que la DAP es heterogénea entre los regantes, variando en función de diversos factores, tales como la aversión al riesgo del agricultor, su determinada exposición al riesgo en función de los cultivos o de circunstancias personales como la edad o el nivel educativo.

Partiendo de esta evidencia empírica, se ha comenzado a desarrollar en la literatura una línea de trabajo centrada en el estudio de un seguro que cubra el riesgo de sequía hidrológica para el regadío. En este sentido, los investigadores Jorge Ruiz, María Bielza, Alberto Garrido y Ana Iglesias ${ }^{43}$, propusieron en España un seguro de ingresos para cubrir el riesgo de sequía hidrológica, con evaluación del daño en la propia explotación mediante peritación, de manera semejante al resto de las líneas del seguro agrario. Este diseño de seguro de daños ha sido, sin embargo, criticado por el carácter sistémico del riesgo de sequía (tanto agrícola como hidrológica) que provoca que, en caso de siniestro, las correspondientes peritaciones resulten difíciles y caras de implementar (exigencia de realizar un gran número de peritaciones al mismo tiempo por técnicos cualificados) ${ }^{44}$. Además de esta debilidad, se señalan como limitaciones de esta propuesta las condiciones del contrato que esta planteaba, que incluían la prohibición de realizar transferencias de agua entre regantes y que limitaba la cobertura del seguro a determinados cultivos (solo aquellos para los cuales la compañía aseguradora pueda calcular el riesgo actuarial).

Frente al seguro agrario tradicional con peritación de daños a cultivos, la literatura ha propuesto como diseño alternativo más eficiente los seguros de sequía indexados. En este tipo de seguros (indexados a una variable), la ocurrencia de siniestro, la medición de la intensidad del mismo y la valoración del daño se realizan de manera indirecta a través de una variable o índice correlacionado con la contingencia cubierta; por ejemplo, la cobertura del riesgo de sequía agrícola en secano puede hacerse operativa de manera simple a través de un índice de precipitaciones acumuladas, sin necesidad de declaraciones individuales de siniestro y peritación de daños parcela por parcela, como en el caso del seguro agrario tradicional. Siguiendo esta idea, los investigadores Kimberly Zeuli y Jerry Skees ${ }^{45}$ diseñaron un contrato de seguro indexado de sequía hidrológica para regadío en

\footnotetext{
${ }^{40}$ World Bank, 2005. OECD, 2011. EC, 2017b.

${ }^{41}$ Garrido y Bardají, 2009.

42 Por ejemplo: Mesa-Jurado et al., 2012.

43 Ruiz et al. 2015.

44 Skees et al., 2008.

${ }^{45}$ Zeuli y Skees, 2005.
}

Australia basado en un índice de precipitaciones. El estudio concluyó evidenciando que el diseño del seguro basado en un índice climatológico tiene muchas ventajas, ya que permite:

a) Disminuir los problemas de riesgo moral y de selección adversa.

b) Compatibilizar el seguro de sequía hidrológica con los mercados de agua.

c) Disminuir los costes del seguro al evitar la necesidad de realizar peritaciones para la cuantificación de las indemnizaciones.

Como principal debilidad de este trabajo, debe destacarse la imperfecta correlación del índice de precipitaciones considerado y el riesgo de sequía hidrológica (fallo en la garantía de suministro).

En Méjico, los investigadores Akssell Leiva y Jerry Skees $^{46}$ propusieron un seguro indexado de sequía hidrológica para regadío tomando como índice la variable "flujos de entrada de agua en los embalses", con dos variantes según la vigencia temporal del contrato: a doce meses y a dieciocho meses. En este trabajo, el diseño del seguro, en cada una de sus dos variantes, se centró en modelizar el cálculo de la prima y la indemnización a recibir por el agricultor.

Otro ejemplo de seguro indexado de sequía hidrológica es el planteado por los investigadores Teresa Maestro, Barry Barnett, Keith Coble, Alberto Garrido y María Bielza ${ }^{47}$ para California. Este trabajo está basado en un índice actualmente ya calculado por la autoridad ambiental del Estado encargada de la gestión del agua con el propósito de estimar la disponibilidad de agua superficial para su reparto entre los regantes. Dicho índice se calcula, cada año, al inicio de la campaña de riego (cada primero de mayo), y estima la disponibilidad de agua en función de la escorrentía en el año en curso y del valor del índice el año anterior. Dado que el valor del índice está afectado por el valor de este mismo índice el año anterior, un seguro basado en el mismo tendría un riesgo potencialmente elevado de selección adversa intertemporal. Para minimizar este problema, estos autores proponen tres diseños alternativos del seguro:

a) Contrato de seguro con contratación temprana (un año antes).

b) Contrato de seguro con prima variable

c) Contrato de seguro con franquicia variable, condicionándose estos dos últimos al valor del índice en el año anterior.

En España, los investigadores Teresa Maestro, María Bielza y Alberto Garrido ${ }^{48}$ han propuesto asimismo un seguro indexado de carácter colectivo (dirigido a comunidades de regantes) para cubrir el riesgo de fallo de suministro en la agricultura de regadío. El seguro permite calcular la indemnización a percibir por el agricultor en base a un índice de sequía hidrológica calculado en función de los flujos de entrada en los embalses. Sin embargo, esta propuesta presenta dos inconvenientes reseñables. Primero, parte de hipótesis hidrológicas muy restrictivas, como por ejemplo que las situaciones de sequía hidrológica no pueden preverse al inicio del año

\footnotetext{
${ }^{46}$ Leiva y Skees, 2008.

47 Maestro et al., 2016a.

${ }^{48}$ Maestro et al., 2016b.
} 
agrícola (los embalses de regulación sólo pueden cubrir las necesidades de un año), circunstancia que difícilmente se da en las cuencas españolas con mayores riesgos de fallo de suministro. Segundo, su carácter colectivo, que dificultaría su implementación práctica al no tener en cuenta la heterogeneidad de las preferencias de los agricultores en relación con la gestión de este riesgo y de los daños ocasionados por los recortes en la dotación (planes de cultivos).

Finalmente cabe reseñar otros trabajos, aunque no han abordado el diseño del seguro considerando toda su complejidad, se han centrado en calcular la prima de un hipotético contrato de seguro, destacando en este sentido los estudios de Carlos Dionisio Pérez-Blanco y Carlos Mario Gómez-Gómez ${ }^{49}$ y el de Dolores Rey, Javier Calatrava y Alberto Garrido ${ }^{50}$.

Propuesta PARA Un CONTRATO DE SEGURO INDEXAdo DE SEQUía HIDROLÓGICA PARA REGADÍO EN ESPAÑA

En este trabajo se propone un seguro indexado de sequía hidrológica para regadío como el tipo de diseño de seguro más adecuado para cubrir este riesgo, dadas sus ventajas sobre los seguros tradicionales. Se considera que los beneficios derivados de los menores costes y la mayor transparencia de este tipo de seguro son superiores a los posibles perjuicios derivados del riesgo base, máxime cuando el índice propuesto está altamente correlacionado con las pérdidas que se quieren asegurar.

Además, otro motivo relevante que justifica la elección de un seguro indexado para cubrir el riesgo de sequía hidrológica frente a un seguro tradicional se fundamenta en el procedimiento seguido en las cuencas hidrográficas españolas para establecer las dotaciones anuales de riego. Efectivamente, aunque existen PES donde se protocoliza la cuantía de las dotaciones en caso de escasez de agua, existe todavía cierto grado de arbitrariedad sobre este aspecto $^{51}$. Esto hace que existan dudas sobre si el fallo de suministro de agua de riego en las cuencas hidrográficas españolas es un evento no manipulable $y$, por tanto, asegurable. Esta circunstancia genera recelos entre las empresas aseguradoras dispuestas a comercializar seguros que cubran este riesgo, lo que justifica el diseño de un seguro indexado que emplee un indicador transparente y no manipulable. Como justifican distintitos investigadores ${ }^{52}$, un índice adecuado en este sentido para un seguro de sequía hidrológica para el regadío podría basarse en el flujo acumulado de entrada de agua en los embalses de regulación o, bien, como es la alternativa elegida en este trabajo, en el "stock de agua disponible en los embalses de regulación”. Además, se propone que el seguro de sequía hidrológica para regadío planteado se incluya, como el resto de las líneas del seguro agrario en España, dentro del SESAC y, por tanto, pueda contar con una subvención parcial de la prima comercial y con un reaseguro público.

El contrato o póliza de seguro es el documento legal donde se establecen las normas que regulan la relación entre asegurador y asegurado, donde se explicita que el

\footnotetext{
49 Pérez-Blanco y Gómez-Gómez, 2013; 2014.

50 Rey et al., 2016b.

51 Pérez-Blanco y Gómez-Gómez, 2013.

52 Brown y Carriquiry, 2007. Leiva y Skees, 2008. Maestro et al., 2016a.
}

primero se obliga, mediante el cobro de una prima y, en el caso de que se produzca el evento objeto de cobertura (siniestro), a indemnizar el daño producido en la forma convenida. En este sentido, a continuación, se presentan los distintos elementos de este contrato que definen las alternativas propuestas para el seguro de sequía en regadío.

En relación con los elementos materiales del contrato, se plantean las siguientes alternativas de diseño:

- Bien asegurado: es el objeto sobre el que recae la cobertura, proponiéndose que en este caso sea la dotación anual de agua superficial concedida por el organismo de cuenca a la explotación asegurada. Se trataría, pues, de una póliza simple, que solo cubriría una garantía concreta. No obstante, aunque esta característica no impediría que este seguro se pudiera suscribir como una cobertura complementaria dentro de las líneas de seguros agrarios tradicionales de las explotaciones de regadío. Para que se pudiera asegurar este riesgo, la explotación no podría tener otras fuentes de agua alternativas para riego (p. ej., pozos).

- Capital asegurado (CA). Se propone que este sea el valor de la dotación anual de agua de riego de la explotación, constituyendo el límite máximo de indemnización que el asegurador estaría obligado a pagar en caso de siniestro total. En este sentido, se plantea que este sea un valor convenido o estimado, equivalente a la diferencia entre el margen bruto anual de la explotación con una dotación de riego normal y el margen bruto anual de la explotación en condiciones de secano. Para la realización de estos cálculos de manera operativa para cada explotación se propone emplear datos tabulados de márgenes brutos medios de los cultivos de regadío y de secano estimados para cada zona regable.

- Índice: es la variable empleada para determinar cuándo ocurre un siniestro y la intensidad de este. Como ya se ha comentado, este índice debe reunir una serie de características deseables entre las que destacan, especialmente, que tenga una elevada correlación con el riesgo asegurado y que sea no manipulable. Teniendo en cuenta estas características y la literatura analizada, se propone que esta variable sea el "stock de agua disponible en los embalses de regulación” del sistema de explotación hidráulico al que pertenece la zona regable del asegurado, que notaremos como SA. De manera más concreta, se propone que este índice se calcule, a fecha 1 de mayo, como la suma del agua almacenada en los embalses de cada sistema al final del último año hidrológico (30 de septiembre del año anterior) más los flujos entrantes en los embalses desde el inicio del año hidrológico (1 de octubre) hasta el 30 de abril del siguiente año. Así, este índice se podría calcular anualmente a primeros de mayo y, en función de su valor, determinar si existe siniestro y, en su caso, la intensidad del mismo, tal y como se comenta a continuación.

- Siniestro: es la manifestación concreta del riesgo asegurado, que se constataría en este seguro 
cuando el valor del índice SA sea inferior a una cantidad determinada previamente (U). Dicha cantidad U se correspondería con el stock de agua mínimo que permitiría al organismo de cuenca aprobar dotaciones de agua equivalentes a los derechos concesionales. Así, la condición para la declaración de un siniestro es que SA sea inferior a U, puesto que se considera que en tales circunstancias se producirán restricciones en las dotaciones de riego y, consecuentemente, un lucro cesante en las explotaciones de regadío. Al tratarse de un riesgo con grado de intensidad variable, el siniestro podría ser parcial (SA inferiores a $U$, pero superiores a $\mathrm{L}$, definido este último parámetro como el stock de agua límite que permite aprobar dotaciones de riego) o total (SA inferiores a L, que provocaría que la dotación anual para riego fuese cero). La declaración de siniestro se propone se realice en todo caso al principio de la campaña de riego, más concretamente el 1 de mayo, una vez se constaten los valores que toma el índice SA.

- Indemnización (I): es el importe que está obligado a pagar contractualmente el asegurador en caso de produzca un siniestro, y que en el seguro propuesto se correspondería con la entrega de una cantidad en metálico equivalente a la estimación de daños ocasionados por el siniestro. Al tratarse de un seguro indexado, el cálculo de la indemnización no requeriría tasación de daños, sino que se calcularía automáticamente al conocerse el valor que toma SA de la siguiente forma:

$$
I=\left\{\begin{array}{ccc}
0 & \text { si } & S A \geq U \\
C A \times(1-F R) \times f(S A) & \text { si } & U>S A>L \\
C A \times(1-F R) & \text { si } & S A \leq L
\end{array}\right.
$$

Como puede apreciarse, la indemnización (I) depende tanto de la franquicia (FR) (porcentaje del capital asegurado que el agricultor asume en forma de autoseguro; esto es, el propio asegurador asume el riesgo) que pudiera acordarse en la póliza, como de la intensidad del siniestro, cuantificado por la función $\mathrm{f}(\mathrm{SA})$.

Como ya se ha comentado, el seguro se regiría por la aplicación del principio indemnizatorio, según el cual el seguro debería reparar únicamente el daño sufrido por los siniestros, evitando en todo caso proporcionar un beneficio al asegurado. En este sentido, para verificar el cumplimiento de este principio, y también con el propósito de abaratar la prima, se propone una franquicia del $30 \%$.

En cuanto a la función que mide la intensidad del siniestro, cabría asumir en principio que esta es lineal:

$$
f(\mathrm{SA})=(\mathrm{U}-\mathrm{SA}) /(\mathrm{U}-\mathrm{L})
$$

Esto implicaría asumir, de manera pragmática, que el valor de la dotación es proporcional a la cantidad de agua asignada.
- Prima: es la aportación económica que tendría que satisfacer el asegurado a la entidad aseguradora en concepto de prestación por la cobertura del riesgo. Se propone una prima anual.

Por lo que respecta a los elementos formales del contrato de seguro, se proponen las siguientes alternativas de diseño:

- Vida del contrato. La vigencia del contrato comenzaría con la formalización de la póliza durante el periodo de contratación establecido (durante el mes de septiembre de cada año) y el correspondiente pago de la prima anual, y terminaría tras el cálculo del índice SA el 1 de mayo del siguiente año. No obstante, la póliza se propone sea prorrogable por anualidades, de forma ilimitada hasta la rescisión del contrato por cualquiera de las partes. Para minimizar la existencia de selección adversa intertemporal, si durante el periodo de contratación se diesen situaciones de prealerta, alerta o emergencia, según se establece en el PES, sólo se permitiría la renovación de las pólizas de años anteriores. La inclusión de nuevos asegurados o modificaciones en los capitales asegurados por cambios en los planes de cultivo sólo sería posible durante el periodo de contratación en años en los que haya una situación hidrológica normal.

- Notificación del siniestro. Al tratarse de un seguro indexado, el asegurado no tendría que comunicar al asegurador la ocurrencia de un siniestro. El día 1 de mayo se constataría el valor del índice SA y, en el caso de que SA fuera menor que $U$, se establecerían las indemnizaciones a recibir por cada asegurado.

Finalmente, en relación con los elementos personales del contrato de seguro se propone:

- Asegurado. Se plantea la posibilidad de suscribir tanto pólizas individuales como pólizas colectivas (p. ej., comunidades de regantes) a través de los correspondientes tomadores.

- Asegurador. Siguiendo el modelo del SESAC, se propone que, al igual que el resto de las líneas del seguro agrario, la línea propuesta para la cobertura de la sequía hidrológica sea asegurada por Agroseguro S.A. Esta empresa aseguradora es de carácter público-privado, y es la única legalmente autorizada en España para suscribir pólizas de seguros agrarios (aunque la comercialización de estas corre a cargo de las diferentes empresas privadas incluidas en el sistema). Así, la propuesta de Agroseguro S.A. como empresa aseguradora implica que la línea de sequía hidrológica goce de las ventajas de participar del SESAC: supervisión pública de los contratos y de las primas, subvenciones a los asegurados y reaseguramiento público.

- Reasegurador. Como el resto de las líneas del seguro agrario incluidas en el SESAC, se propone que el seguro indexado propuesto se reasegure públicamente por el Consorcio de Compensación de Seguros. 
ANÁLISIS ACTUARIAL DEL CONTRATO DE SEGURO DE SEQUía HIDROLÓGICA PROPUESTO

Una vez planteada la discusión sobre qué tipo de seguro sería el más adecuado para cubrir el riesgo de sequía hidrológica para regadío y cómo se podrían caracterizar todos sus elementos q, es necesario llevar a cabo un análisis actuarial al objeto de calcular la prima comercial del seguro. Así, este apartado plantea en qué debería consistir el trabajo futuro (objetivos concretos del análisis) y qué métodos deberían emplearse al objeto de que el SESAC pudiese ofrecer a los regantes este tipo de seguro.

La primera etapa consistirá en confirmar, para cada sistema de explotación, que el índice propuesto (SA) está altamente correlacionado con la ocurrencia y la intensidad de los fallos de suministro (dotación de riego inferior a concesión administrativa) y, por tanto, con las pérdidas ocasionadas en las explotaciones de regadío, utilizando para ello las correspondientes técnicas estadísticas de correlación y regresión.

Una segunda etapa requerirá la realización de estudios actuariales sobre los riesgos a asegurar, al objeto de determinar la prima comercial del seguro, que permita a Agroseguro S.A. cubrir las pérdidas esperadas en concepto de indemnizaciones por siniestros, los costes de gestión (administrativos, de comercialización y de tasación), así como los impuestos y recargos (reaseguro), debiendo proporcionar, además, un beneficio mínimo a esta empresa público-privada.

El punto de partida de la prima comercial es el cálculo de la prima base (PB), que representa la esperanza matemática de las indemnizaciones a pagar por los siniestros, teniendo en cuenta las fórmulas de valoración de las indemnizaciones (capital asegurado, franquicia y función de intensidad del siniestro):

$$
\mathrm{PB}=\mathrm{E}(\text { Indemnizaciones })
$$

Este cálculo requiere estimar la función de densidad de probabilidad de las indemnizaciones a partir de simulaciones basadas en el historial de los valores del índice y la fórmula de valoración de las indemnizaciones ${ }^{53}$.

Para reflejar el coste real del riesgo asumido por el asegurador, la $\mathrm{PB}$ se debe incrementar con los gastos relacionados con la peritación de los siniestros (nulos en el caso del seguro indexado propuesto) y con la gestión interna de la tramitación de los siniestros (gastos de prestaciones, Gp), dando lugar a la prima de riesgo neta (PRn):

$$
\mathrm{PRn}=\mathrm{PB}+\mathrm{Gp}
$$

Esta PRn necesita ser aumentada, a su vez, con la prima del reaseguro del Consorcio de Compensación de Seguros (Pccs), llegándose así al cálculo de la prima de riesgo (PR):

$$
\mathrm{PR}=\mathrm{PRn}+\mathrm{Pccs}
$$

Esta PR se requiere incrementar de nuevo con un "recargo se seguridad" cuando la probabilidad de ocurrencia de los siniestros no esté perfectamente caracterizada (como en el caso del seguro propuesto, dado que la serie histórica del índice está afectada por los efectos del cambio climático), o cuando el riesgo asegurado tenga un carácter sistémico (también como en el caso del seguro propuesto). En el primer caso, la PR se incrementa con un "recargo de ambigüedad" $(\alpha)$, mientras que en el segundo se incrementa con un "recargo de catástrofe" $(\lambda)$. El resultado de esta prima incrementada equivale a la prima de riesgo recargada (PRR):

$$
\mathrm{PRR}=\mathrm{PR}+\alpha \cdot \mathrm{PRn}+\lambda \cdot \mathrm{PRn}
$$

A la PRR se le debe añadir los gastos internos de gestión y administración (G) de Agroseguro S.A., como única compañía aseguradora que puede ofertar la línea propuesta dentro del SESAC, resultando así la Prima comercial base del coaseguro (PCb):

$$
\mathrm{PCb}=\mathrm{PRR}+\mathrm{G} \cdot \mathrm{PCb}=\frac{\mathrm{PRR}}{(1-\mathrm{G})}
$$

Asimismo, a esta última se le deberán añadir los gastos externos de gestión de las entidades coaseguradoras (G') para obtener la Prima Comercial (PC):

$$
P C=P C b+G^{\prime} \cdot P C=\frac{P C b}{\left(1-G^{\prime}\right)}
$$

Finalmente, la prima comercial se incrementa con los gravámenes complementarios que procedan, tales como impuestos, y se reducirá por las subvenciones que pudieran existir por la suscripción del seguro, resultando finalmente el recibo de la prima, que coincide con la cantidad a pagar por el asegurado.

En la realización de este análisis actuarial requerirán una especial atención:

- La estimación de las funciones de densidad de probabilidad de las indemnizaciones, que se obtendrán a partir de la información histórica disponible sobre los datos climáticos e hidrológicos de los diferentes sistemas de explotación hidrográficos donde se localicen los potenciales asegurados.

- El coste del reaseguro, para lo que será necesario analizar los costes actualmente cargados al reaseguramiento obligatorio de los seguros agrarios por parte del Consorcio de Compensación de Seguros.

- La cuantía de los recargos por ambigüedad y catástrofe, que se establecerían aplicando técnicas financieras específicas.

El resto de los datos necesarios para la determinación de la prima total (costes de administración, costes de comercialización o beneficio industrial) se podría obtener a partir de coeficientes técnicos normalmente empleados por la industria del seguro para el sector agrario.

\footnotetext{
53 Zeuli y Skees, 2005. Brown y Carriquiry, 2007. Leiva y Skees, 2008. Maestro et al., 2016b.
} 


\section{CONCLUSIONES}

Según se ha constatado, el fallo en la garantía de suministro del agua de riego es un tipo de riesgo cada vez más relevante en el regadío de las regiones de clima mediterráneo o semi-árido, como son las zonas del sur y este peninsular español. La importancia de este riesgo deriva no sólo de las graves consecuencias que este conlleva (principalmente disminuciones de renta del agricultor), sino también por el hecho de que, en el actual escenario de cambio climático, se vislumbra una mayor incidencia de sequías hidrológicas y fallos en la garantía de suministro.

La relevancia creciente de este riesgo de sequía obliga, por tanto, a debatir acerca de su gestión, así como a proponer y analizar nuevos instrumentos para minimizar sus impactos. En este sentido, se ha constatado que los instrumentos de oferta de la tradicional política de agua basada en la construcción de infraestructuras ya no son opciones viables en la mayoría de las regiones con economías del agua maduras. Como alternativa, se plantea el desarrollo de nuevos instrumentos de demanda, como podría ser el seguro de sequía hidrológica. De esta forma, el seguro de sequía hidrológica aparece como un nuevo instrumento de gestión de la demanda de agua alternativo a otros que han evidenciado resultados dispares, tales como la modernización de regadíos o los mercados de agua.

En este artículo se ha propuesto un seguro de sequía hidrológica de carácter indexado a través de la variable "stock de agua disponible en los embalses de regulación" para cubrir el fallo en la garantía de suministro en regadío. Este nuevo instrumento sería una alternativa viable, ya que permitiría minimizar muchos de los problemas o factores limitantes de los tradicionales seguros agrarios, como los de selección adversa o de riesgo moral, y minimizaría el coste de la prima por no requerir de tasación de los daños a los cultivos mediante peritación. El seguro indexado propuesto podría, además, solventar ciertas limitaciones propias de la complejidad de aseguramiento del riesgo de sequía. Entre ellas, cabría destacar las derivadas de la arbitrariedad que aún existe en el procedimiento seguido a la hora de establecer las dotaciones anuales de agua por las comisiones de desembalse. Asimismo, el seguro indexado propuesto permitiría la conjunción su implementación conjunta con otros instrumentos de gestión del riesgo, como puede ser la cesión temporal de derechos a través de los centros de intercambio (bancos de agua) o contratos de cesión (mercados de agua).
La implementación de la propuesta de seguro realizada requiere de estudios adicionales desde la perspectiva de la oferta, al objeto de calcular la prima comercial del seguro mediante los correspondientes métodos actuariales y financieros, y desde la perspectiva de la demanda, para conocer la potencial aceptación (disposición a pagar -DAP- por contratar este instrumento) por parte de los regantes. La comparación de los resultados de estos estudios de oferta y de demanda permitirán conocer la viabilidad comercial del seguro planteado. Si, para una mayoría de regantes, la DAP es mayor que la prima comercial, entonces el seguro sería viable sin necesidad de subvenciones, ya que la decisión de esta mayoría de productores sería la contratación del seguro. En cualquier caso, siempre habrá un porcentaje de regantes para los cuales la DAP sea menor que la prima comercial y, por tanto, no contrate el seguro propuesto. En este sentido, el establecimiento de subvenciones públicas, como en el resto de las líneas del SESAC, produce un abaratamiento de la prima comercial para los regantes, ya que el desembolso que tendrían que hacer sería equivalente a la diferencia entre la prima comercial y la subvención recibida. De esta manera, a medida que la subvención a la contratación del seguro se incremente, habrá un porcentaje creciente de regantes que opten por suscribirlo. Mediante la realización de un análisis de la heterogeneidad de las preferencias de los individuos se podrá establecer a priori el nivel de implantación del seguro propuesto para cualquier nivel de subvención. Esta información resultará clave para soportar la decisión política sobre el nivel de apoyo con que debe contar esta nueva línea. Estos estudios fundamentan las futuras líneas de investigación a desarrollar con el propósito de poner en práctica este nuevo seguro, y el consecuente logro del objetivo de mejorar el desempeño económico, social y ambiental del regadío, contribuyendo positivamente a su resiliencia en el actual escenario de cambio climático. 


\section{BiBliografíA}

Agroseguro, S.A. 2018: El seguro agrario en cifras 2017. Madrid, Agroseguro.

Antón, J. 2009: "Políticas agrarias y gestión de riesgos: una aproximación global”, Revista Española de Estudios Agrosociales y Pesqueros, 221, 71-94.

Antón, J. y Kimura, S. 2011: Risk Management in Agriculture in Spain. Paris, OECD Publishing.

Bardají, I., Escribano, S. y Garrido, A. 2016: Principios básicos de seguros agrarios. Almería, Cajamar Caja Rural.

Beare, S. C., Bell, R. y Fisher, B. S. 1998: "Determining the value of water: The role of risk, infrastructure constraints, and ownership”, American Journal of Agricultural Economics, 80(5), 916-940. http://dx.doi.org/10.2307/1244183

Berbel, J. y Gutiérrez-Martín, C. 2017: Efectos de la modernización de regadíos en España. Almería, Cajamar Caja Rural.

Bielza, M.; Conte, C. G.; Catenaro, R. y Gallego-Pinilla, F. J. 2008: Agricultural Insurance Schemes II. Index Insurances. Ispra (Italy), Joint Research Centre.

Borch, K. H. 1990: Economics of Insurance. Amsterdam, North-Holland.

Brown, C. y Carriquiry, M. 2007: "Managing hydroclimatological risk to water supply with option contracts and reservoir index insurance”, Water Resources Research, 43(11), 1-13. http://dx.doi.org/10.1029/2007WR006093

Burgaz, F. J. 1995: "Las políticas de ayudas a las catástrofes agrícolas y a los seguros agrarios en la Unión Europea”, Revista Española de Economía Agraria, 174, 289-308.

Burgaz, F. J. y Pérez-Morales, M. M. 1996: 1902-1992: 90 años de seguros agrarios en España. Madrid, Ministerio de Agricultura, Pesca y Alimentación.

Coble, K. H.; Knight, T .O.; Pope, R. D. y Williams, J.R. 1997: “An expected-indemnity approach to the measurement of moral hazard in crop insurance”, American Journal of Agricultural Economics, 79(1), 216-226. http://dx.doi.org/10.2307/1243955

EC (European Commission) 2017a: Communication from the Commission to the European Parliament, the Council, the European Economic and Social Committee and the Committee of the Regions. The future of food and farming. Brussels, European Commission.

EC (European Commission) 2017b: Risk management schemes in EU agriculture. Dealing with risk and volatility. Brussels, European Commission.

Expósito, A. y Berbel, J. 2017: “Agricultural irrigation water use in a closed basin and the impacts on water productivity: The case of the Guadalquivir river basin (Southern Spain)”, Water, 9(2), 1-15. http://dx.doi.org/10.3390/w9020136

Gallego-Ayala, J.; Gómez-Limón, J. A. y Arriaza, M. 2011: "Irrigation water pricing instruments: A sustainability assessment”, Spanish Journal of Agricultural Research, 9(4), 981-999. http://dx.doi.org/10.5424/sjar/20110904-449-10

Garrido, A. y Bardají, I. 2009: “Estrategias para la gestión de riesgos y crisis en la agricultura española”, Revista Española de Estudios Agrosociales y Pesqueros, 221, 175-205.

Garrido, A. y Gómez-Ramos, A. 2009: "Risk management instruments supporting drought planning and policy”, en Iglesias, A., Garrote, L., Cancelliere, A., Cubillo, F. y Wilhite, D.A. (eds): Coping with Drought Risk in Agriculture and Water Supply Systems. Drought Management and Policy Development in the Mediterranean, Springer, Dordrecht (The Netherlands), 133-151.

Gómez-Limón, J. A. y Picazo-Tadeo, A. J. 2012: "Irrigated agriculture in Spain: Diagnosis and prescriptions for improved governance", International Journal of Water Resources Development, 28(1), 57-72. http://dx.doi.org/10.1080/07900627.2012.640876

IPCC 2014: Climate Change 2014: Impacts, Adaptation, and Vulnerability. Part A: Global and Sectoral Aspects. Contribution of Working Group II to the Fifth Assessment Report of the Intergovernmental Panel on Climate Change. Cambridge (UK), Cambridge University Press.
Jensen, N. D. y Barrett, C. B. 2017: “Agricultural index insurance for development”, Applied Economic Perspectives and Policy, 39(2), 199219. http://dx.doi.org/10.1093/aepp/ppw022

Just, R. E.; Calvin, L. y Quiggin, J. 1999: “Adverse selection in crop insurance: Actuarial and asymmetric information incentives”, American Journal of Agricultural Economics, 81(4), 834-849. http://dx.doi.org/10.2307/1244328

Lago, M.; Mysiak, J.; Gómez, C. M.; Delacámara, G. y Maziotis, A. 2015: Use of Economic Instruments in Water Policy: Insights from International Experience. Cham (Switzerland), Springer.

Leiva, A. J. y Skees, J. R. 2008: "Using irrigation insurance to improve water usage of the Rio Mayo Irrigation System in Northwestern Mexico”, World Development, 36(12), 2663-2678. http://dx.doi.org/10.1016/j.worlddev.2007.12.004

Machetti, I. 2015: "El sistema de seguros agrarios combinados en España”, Consorseguros, 2, 1-22.

Maestro, T.; Barnett, B. J.; Coble, K.H.; Garrido, A. y Bielza, M. 2016a: "Drought index insurance for the Central Valley Project in California", Applied Economic Perspectives and Policy, 38(3), 521-545. http://dx.doi.org/10.1093/aepp/ppw013

Maestro, T.; Bielza, M. y Garrido, A. 2016b: "Hydrological drought index insurance for irrigation districts in Spain”, Spanish Journal of Agricultural Research, 14(3), 1-14. http://dx.doi.org/10.5424/sjar/2016143-8981

Marques, G. F.; Lund, J. R. y Howitt, R. E. 2005: “Modeling irrigated agricultural production and water use decisions under water supply uncertainty”, Water Resources Research, 41(8), 1-11. http://dx.doi.org/10.1029/2005WR004048

Menapace, L.; Colson, G. y Raffaelli, R. 2013: "Risk aversion, subjective beliefs, and farmer risk management strategies”, American Journal of Agricultural $\quad$ Economics, 384-389. http://dx.doi.org/10.1093/ajae/aas107

Mesa-Jurado, M.A.; Martin-Ortega, J.; Ruto, E. y Berbel, J. 2012: “The economic value of guaranteed water supply for irrigation under scarcity conditions”, Agricultural Water Management, 113, 10-18. http://dx.doi.org/10.1016/j.agwat.2012.06.009

Meuwissen, M. P. M.; de Mey, Y. y van Asseldonk, M. 2018: "Prospects for agricultural insurance in Europe”, Agricultural Finance Review, 78(2), 174-182. http://dx.doi.org/10.1108/AFR-04-2018-093

Mishra, A. K; Wesley Nimon, R. y El-Osta, H. S. 2005: "Is moral hazard good for the environment? Revenue insurance and chemical input use", Journal of Environmental Management, 74(1), 11-20. http://dx.doi.org/10.1016/j.jenvman.2004.08.003

Montilla-López, N. M.; Gutiérrez-Martín, C. y Gómez-Limón, J. A. 2016: "Water banks: What have we learnt from the international experience?”, Water, 8(10), 1-19. http://dx.doi.org/10.3390/w8100466

Moschini, G. y Hennessy, D. A. 2001: "Uncertainty, risk aversion, and risk management for agricultural producers”, en Gardner, B. L. y Rausser, G. C. (eds): The Handbook of Agricultural Economics, Elsevier Science, Amsterdam (The Netherlands), 87-153.

OECD (Organisation for Economic Co-operation and Development) 2011: Managing Risk in Agriculture: Policy Assessment and Design. Paris, OECD Publishing.

OECD (Organisation for Economic Co-operation and Development) 2016: Mitigating Droughts and Floods in Agriculture. Policy Lessons and Approaches. Paris, OECD Publishing.

Palomo-Hierro, S. y Gómez-Limón, J. A. 2013: “El papel de los mercados como instrumento para la reasignación del agua en España”, Agua y Territorio, 2, 78-92. http://dx.doi.org/10.17561/at.v1i2.1347

Palomo-Hierro, S.; Gómez-Limón, J. A. y Riesgo, L. 2015: “Water markets in Spain: Performance and challenges", Water, 7(2), 652-678. http://dx.doi.org/10.3390/w7020652 
Pérez-Blanco, C. D. y Gómez-Gómez, C. M. 2013: "Designing optimum insurance schemes to reduce water overexploitation during drought events: a case study of La Campiña, Guadalquivir River Basin, Spain”, Journal of Environmental Economics and Policy, 2(1), 1-15. http://dx.doi.org/10.1080/21606544.2012.745232

Pérez-Blanco, C. D. y Gómez-Gómez, C. M. 2014: "Insuring water: a practical risk management option in water-scarce and drought-prone regions?”, Water Policy, 16(2), 244-263. http://dx.doi.org/10.2166/wp.2013.131

Rey, D.; Calatrava-Leyva, J. y Garrido, A. 2016a: “Optimisation of water procurement decisions in an irrigation district: The role of option contracts", Australian Journal of Agricultural and Resource Economics, 60(1), 130-154. http://dx.doi.org/10.1111/1467-8489.12110

Rey, D.; Garrido, A. y Calatrava-Leyva, J. 2016b: “Comparison of different water supply risk management tools for irrigators: Option contracts and insurance”, Environmental and Resource Economics, 65(2), 415-439. http://dx.doi.org/10.1007/s10640-015-9912-2

Rey, D.; Pérez-Blanco, C. D.; Escriva-Bou, A.; Girard, C. y Veldkamp, T. I. E. 2019: "Role of economic instruments in water allocation reform: lessons from Europe", International Journal of Water Resources Development, 35(2), 206-239. http://dx.doi.org/10.1080/07900627.2017.1422702
Riera, C. 2018: “Agua subterránea y riego mecanizado: distinción y vulnerabilidad social ante el riesgo de sequía en Río Segundo, Córdoba, Argentina”, Agua y Territorio, 12, 119-132. http://dx.doi.org/10.17561/at.12.3338

Ruiz, J.; Bielza, M.; Garrido, A. y Iglesias, A. 2015: "Dealing with drought in irrigated agriculture through insurance schemes: An application to an irrigation district in Southern Spain”, Spanish Journal of Agricultural Research, 13(4), 1-15. http://dx.doi.org/10.5424/sjar/2015134-6941

Skees, J. R.; Barnett, B. J. y Collier, B. 2008: Agricultural insurance. Background and context for climate adaptation discussions. Lexington (Kentuvky-USA), GlobalAgRisk, Inc.

World Bank 2005: Managing Agricultural Production Risk: Innovations in Developing Countries. Washington, D.C., World Bank.

World Bank 2011: Weather index insurance for agriculture: Guidance for development practitioners. Washington, D.C., World Bank.

Zeuli, K. A. y Skees, J. R. 2005: "Rainfall insurance: A promising tool for drought management”, International Journal of Water Resources Development, $21(4)$, http://dx.doi.org/10.1080/07900620500258414 